\title{
Special Issue on Admissible Rules and Unification
}

\author{
Rosalie lemhoff and George Metcalfe
}

Admissible rules play a sometimes implicit but fundamental role in many fields of mathematics and computer science. Algorithmically, they can be described as rules that when added to a system produce no new theorems, and semantically, as rules where if the premises of an instance are theorems, then so is the conclusion. The study of admissible rules spans a broad range of topics, including free and projective algebras, equational unification, duality, and proof theory.

The notion of an admissible rule was defined explicitly by Paul Lorenzen in the 1950 s in the context of intuitionistic logic, but had appeared already in earlier work of Gerhard Gentzen, Ingebrigt Johansson, and others. The closely related topic of universal sentences valid in free algebras also played a prominent role in the work of Phillip Whitman and Bjarni Jónsson on free lattices. Research on admissible rules initially focused mainly on intuitionistic logic, culminating in Vladimir Rybakov's proof that its set of admissible rules is decidable but not finitely axiomatizable, and independent completeness proofs by Rosalie Iemhoff and Paul Rozière for an elegant axiomatization conjectured by Dick De Jongh and Albert Visser. Iemhoff's proof stems from Silvio Ghilardi's pioneering work on equational unification, which has provided the springboard for a resurgence of interest in admissible rules and their relationship to unification. The papers in this volume bear witness both to this resurgence and its broad scope.

The paper Admissible rules of the Leibniz hierarchy by James Raftery presents an analysis of admissibility in the framework of abstract algebraic logic. The key achievement of this work is to extend the reach of the main ideas and theorems for admissible rules, typically formulated algebraically, to nonalgebraizable logics of the "Leibniz hierarchy." These methods are put to work to obtain new results for admissibility in certain substructural logics. Related substructural logics are investigated in the paper An Abelian rule for $\mathrm{BCl}$ - and variations by Tomasz Kowalski and Lloyd Humberstone. The authors provide an ingenious proof-theoretic argument to show the admissibility of certain nonderivable rules and hence the failure of structural completeness for these logics. 


\title{
Special Issue on Admissible Rules and Unification
}

\author{
Rosalie lemhoff and George Metcalfe
}

Admissible rules play a sometimes implicit but fundamental role in many fields of mathematics and computer science. Algorithmically, they can be described as rules that when added to a system produce no new theorems, and semantically, as rules where if the premises of an instance are theorems, then so is the conclusion. The study of admissible rules spans a broad range of topics, including free and projective algebras, equational unification, duality, and proof theory.

The notion of an admissible rule was defined explicitly by Paul Lorenzen in the 1950 s in the context of intuitionistic logic, but had appeared already in earlier work of Gerhard Gentzen, Ingebrigt Johansson, and others. The closely related topic of universal sentences valid in free algebras also played a prominent role in the work of Phillip Whitman and Bjarni Jónsson on free lattices. Research on admissible rules initially focused mainly on intuitionistic logic, culminating in Vladimir Rybakov's proof that its set of admissible rules is decidable but not finitely axiomatizable, and independent completeness proofs by Rosalie Iemhoff and Paul Rozière for an elegant axiomatization conjectured by Dick De Jongh and Albert Visser. Iemhoff's proof stems from Silvio Ghilardi's pioneering work on equational unification, which has provided the springboard for a resurgence of interest in admissible rules and their relationship to unification. The papers in this volume bear witness both to this resurgence and its broad scope.

The paper Admissible rules of the Leibniz hierarchy by James Raftery presents an analysis of admissibility in the framework of abstract algebraic logic. The key achievement of this work is to extend the reach of the main ideas and theorems for admissible rules, typically formulated algebraically, to nonalgebraizable logics of the "Leibniz hierarchy." These methods are put to work to obtain new results for admissibility in certain substructural logics. Related substructural logics are investigated in the paper An Abelian rule for $\mathrm{BCl}$ - and variations by Tomasz Kowalski and Lloyd Humberstone. The authors provide an ingenious proof-theoretic argument to show the admissibility of certain nonderivable rules and hence the failure of structural completeness for these logics. 